\title{
Results and Performance Analysis of Local Broadcast Algorithms in Wireless Ad hoc Networks: Reducing the Number of Transmissions
}

\author{
Rathi Varsha G. \\ PG Student, \\ MBES College of Engineering, Ambajogai
}

\author{
V.R. Chirchi \\ Assistant Professor, \\ PG Dept, MBES COE, \\ Ambajogai
}

\begin{abstract}
Among Many fundamental communication primitives in wireless ad hoc network, flooding in wireless ad hoc network reduces the number of transmission in network and it plays a significant role in which every node transmits the message after receiving it for the first time. To successfully reducing the number of transmissions of broadcast that are required to achieve full delivery and constant approximation to the optimum solution without using exact position information of node. This paper presents two main approaches such as static and dynamic to broadcast algorithm in wireless ad hoc network. In static approach node status depends on local topology and priority function of node using this approach it can't guarantee both full delivery and constant approximation if node exact position information isn't available. On other hand in dynamic approach if node exact position information is available or not they can achieve both full delivery and constant approximation to their optimum solution using their partial 2-hop hybrid algorithm.
\end{abstract}

\section{Keywords}

Ad hoc network, Broadcasting, Connected Dominating Set (CDS), Constant Approximation.

\section{INTRODUCTION}

Wireless networks consist of a number of nodes which communicate with each other over a wireless channel which have various types of networks: sensor network, ad-hoc mobile networks, cellular networks and satellite networks. Wireless ad-hoc network are collections of wireless nodes that communicate directly over a common wireless channel. The network is an ad hoc means wireless network without infrastructure, they can be called spontaneous network and establishment of this network must be in distributed manner. The main problems in ad hoc networks such as routing in wired networks in infrastructure's networks a node can communicate with all nodes in the same cell. In ad hoc network a node can communicate only with nodes in its area. Ad-hoc networks can use flooding for forwarding data flooding is well suited for MANETs as it requires no topological knowledge. It consists in each node rebroadcasting a message to its neighbors upon receiving it for the first time. Although straightforward, flooding is far from optimal and generates a high number of redundant transmissions of message, wasting limited valuable resources such as bandwidth and energy supplies [7].

This paper is organized as follows: In section II, Literature Survey of paper. In section III, explained proposed method. In. section IV, results and discussion in section V, conclusion and future scope.

\section{LITERATURE SURVEY}

Numbers of flooding algorithms have been proposed in past for broadcasting some are explaining as follows:

- Broadcasting is a familiar operation in a network to solve many issues such as flooding. In flooding high number of redundant transmission occur by causing redundancy, contention, and collision. If this flooding is done blindly in a network. So conjointly we refer to this problem called as a broadcast storm problem. To solve this problem the redundant transmission of message is reduced by applying several schemes such as probabilistic, counter-based, distance-based, location-based, and cluster-based schemes [1].

- Flooding in wireless ad-hoc network has leads to serious redundancy, contention and collision when every node rebroadcast a message that it was received for first time and consumes network resources such as large number of copied messages. To solve this problem a new broadcast approach has been proposed to reduce broadcast redundancy in wireless ad hoc network they use local topology information and statistical information of copied message to avoid rebroadcast of message. And a new broadcast approach can be divided into two parts such as local neighborhood discovery and data broadcasting. In local neighborhood discovery nodes can exchange a hello message by using neighbor information of hello message and data broadcasting source node just broadcast message to its entire neighbor and ignore copied message received later [5].

- Using the plain flooding algorithm causes a high number of unnecessary rebroadcasts message and wasting its number of resources. To solve this problem one more algorithm defined on the basis of probabilistic flooding algorithm such as percolation theory and random graphs. Percolation theory have been applied to wireless networks in that source node broadcast the message with probability one after receiving the message. Node $n$ rebroadcast the message with probability $p_{n}$ and discards with probability $1-\mathrm{p}_{\mathrm{n}}$. Second algorithm is random graph a random graph is a graph where the number of nodes, edges and connections between them are determined in some random manner [2].

- Y. CAI, K. Hua proposed a flooding technique called as edge forwarding for its one hop neighbor. In this technique flooding traffic reduces by using each node broadcast message for its only one hop neighbor distance [6].

- H. Liu, P. Wan proposed on 1-hop neighbor's information with two conditions such as the number of forwarding node is minimal and time complex city of forwarding node is low [3]. 
- Majid Khabbazian proposed two efficient broadcast algorithms based on one hop neighbor information one is sender- based algorithm and a receiver-based algorithm. In sender-based algorithm node selects subset of its neighbors to forward message and receiver-based algorithm receiver decides whether or not to forward message [4].

\section{PPROPOSED SYSTEM}

In past proposed methods has some problem to remove these problems, in this paper a method for flooding reduces the number of transmission has been proposed.

Proposed system mainly divides into two approaches:

\subsection{Static Approach}

In the static approach status of each node proactively based on local topology information and global known priority function, if this local topology changes it can affect status of each node in the area near. Using priority function and local topology information they can't guarantee good approximation factor to the optimum solution if node position information isn't available. If node position information is available it can achieve interesting result such as a constant approximation factor and shortest path preservation.

\subsection{Dynamic Approach}

Drawback of static approach is that all nodes have been static \& data loss problem is occurred to avoid this problem dynamic approach uses. In dynamic approach status of each node is on the fly basis of local topology information and broadcast state information. If local topology changes it can't affect on status of node. In this approach it uses two algorithms such as neighbor-designating and self-pruning. In neighbor-designating each node forward the message to its neighbor using 1-hop neighbor information but in self-pruning algorithm every node decides itself to forward message or not and they use 2-hop neighbor information.2-hop neighbor information has two rounds of information exchange. In the first round request should send with its id number and in second round neighbor node information is send with its id number to its id requesters. On the other hand local algorithm based on dynamic approach can achieve full delivery and constant approximation if exact node position information isn't available and also they can achieve optimum solution if selected node has to broadcast message even if its coverage condition is satisfied.

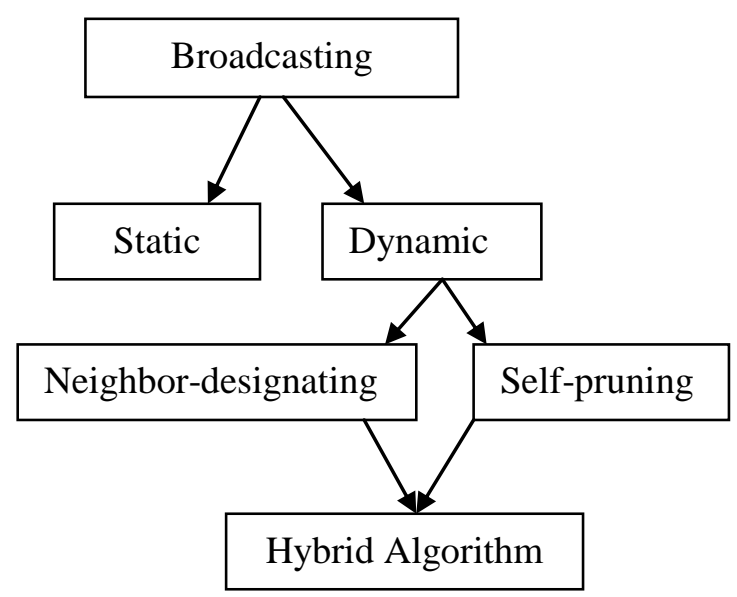

In figure 1 dynamic approach uses two algorithms such as neighbor-designating and self-pruning the combination of this algorithm is called as a hybrid algorithm for 2-hop neighbor information exchange. The proposed hybrid algorithm executed by following steps such as

1. search id's of node

2. select node(id)

3. if (node(id)!=already exist) then

4. create(list)

5. else

6. discard

7. if $(m=1)$ then

8. create and fill list

9. else

10. discard the message

11. Update the list

12. Remove the previous broadcast $(\mathrm{m})$ from list

13. select $\mathrm{id}(\mathrm{m})$ from the list

14. add id(m) then

15 . schedule the message

16. if already message in the queue

17. discard

18. else

19. forward

The proposed algorithm is a hybrid algorithm to broadcast node in its neighbor area in that it first search id of node and then create list of node otherwise node id is already exists then it will be discard that node id. The node id is used to find the place of destination node. After receiving the message, first time a node has to be creating the coverage area with its id number of neighboring nodes. For example, a node u sends the id number to the neighboring node $v$. If the message is received from its neighboring node $\mathrm{v}, \mathrm{v}$ node does not select the node $u$ and send message to node $w$. Note that the node $w$ may not be a neighbor of node $u$. However, since the w node is a neighbor of $v$ node, it is at most 2 hops away from u node. Having id's of $\mathrm{v}$ and $\mathrm{w}$ node are stored in the coverage area details. Since the $\mathrm{w}$ node will eventually broadcast the message, by updating the list, the $\mathrm{u}$ node removes those neighbors that have received the message or will receive it, eventually. Every time the u node receives a copy of message and it will be updated in the list of that node. If the u node is selected by the $\mathrm{v}$ node to send the message, the neighbors of $\mathrm{v}$ node is deleted in the list of $u$ node. Because the $u$ node has to be update the present level to avoid the waste transition.

When $u$ node receives a message, it creates a list and updates the list and select id of node from list as explained earlier. When message is selected to forward if its coverage condition is satisfied and schedule copy of that message in its Medium Control Access (MAC) layer queue. There are at least two sources of delay in the MAC layer. First one is copy of message is already in queue it does not place message in queue. Second one is remove the message from queue if this message has been already selected from queue. On other hand when message is not selected to forward if it's coverage area is not satisfied then creating list of message is becomes empty.

Fig 1: Abstract Diagram 


\section{RESULTS AND DISCUSSION}

The proposed approach is implemented in Network Simulator (NS2) software. The parameters used for comparison between Two-ray ground and shadowing are the ratio of broadcasting nodes, transmission range, average delay, maximum speed. All these parameters are analyzed with network simulator and their performances are presented in Figure 2 to Figure 5 and the analysis of all these figures is summarized in Table 2. Simulation parameters used are listed in Table 1 for quick reference.

Table 1: Simulation Parameters

\begin{tabular}{|c|c|}
\hline Parameter & Value \\
\hline Simulator & Ns-2(version 2.32) \\
\hline MAC Layer & IEEE 802.11 \\
\hline Propagation Model & Two-ray ground \\
\hline Packet Size & 2000 bytes \\
\hline Area & $1000 \mathrm{~m} \mathrm{X} \mathrm{1000} \mathrm{m}$ \\
\hline Bandwidth & $2 \mathrm{Mb} / \mathrm{sec}$ \\
\hline Average Forwarding Delay & $1 \mathrm{~ms}$ \\
\hline Transmission Range & $250 \mathrm{~m}$ \\
\hline Maximum Speed & $20 \mathrm{~m} / \mathrm{s}$ \\
\hline Number of Nodes & $50-150$ \\
\hline
\end{tabular}

To calculate the number of broadcasting nodes, network is consistently dividing the nodes in square area of size $1000 \mathrm{~m} \mathrm{x}$ $1000 \mathrm{~m}$. Then we use only one broadcast at each simulation run. Fig. 2 shows ratio of broadcasting nodes vs. number node. It is defined as the ratio of number packets received by the destination to the number of packets originated by the source.

This ratio is calculated by formula such as

Ratio of broadcasting node $=$ No. of Nodes Broadcast the packet/Total Number of Nodes in Network

This ratio having better performance if they have greater value for broadcasting nodes and this ratio is increases in terms of shadowing and decreases in terms of two- ray round. Fig 2 shows ratio of broadcasting nodes vs. number node on $\mathrm{X}$-axis number of nodes and $\mathrm{Y}$-axis ratio of broadcast nodes.
In fig. 3, shows set of transmission range 100 to $300 \mathrm{~m}$ and numbers of node are between 50-150. The transmission range is selected from a large interval so that simulation covers all nodes in the network. Transmission range means if there are three nodes are presented at the same place, then transmission range based on the node will be choose. Transmission range depends on mobility prediction and node continuation. Mobility Prediction depends on the nodes estimation of their future locations and node continuation means previous location of node. Transmission range is calculated by given formula such as

Transmission Range=Transmission Range/ No. of Node Broadcast the Packet

This ratio is increases in terms of shadowing and decreases in terms of two ray ground. Fig 3 shows transmission range vs. ratio of broadcasting node on $\mathrm{X}$-axis is the transmission range and $\mathrm{Y}$-axis is the ratio of broadcasting nodes.

In fig. 4 shows, one more factor called average delay; this average delay is the average time it takes a data packet to reach the destination. This includes all possible delays caused by buffering during route discovery latency, queuing at the interface queue. This is the average overall delay for a packet to traverse from a source node to a destination node. This includes the route discovery time, the queuing delay at a node, the transmission delay at the MAC layer and the propagation and transfer time in the wireless channel. Average delay is calculated by given formula such as

$$
\text { Avg. Delay }=\mathrm{S} / \mathrm{N}
$$

Where $S$ is the sum of the time spent to deliver packets for each destination, and $N$ is the number of packets received by the all destination nodes. This ratio is increases in terms of shadowing and decreases in terms of two ray ground. Fig 4 shows number of nodes vs. end to end delay on $\mathrm{X}$-axis is the number of nodes and $\mathrm{Y}$-axis is the end to end delay.

In fig. 5 shows ratio of broadcasting nodes versus maximum speed the maximum speed is set in random waypoint mobility model as the number of transmissions slightly decreases mobility of node increases. Maximum speed is calculated by given formula such as

Speed =Maximum speed in Network/Number of Broadcasting Nodes

This ratio increases in terms both shadowing and two ray ground. Fig 5 shows ratio of broadcasting nodes vs. maximum speed on $\mathrm{X}$-axis is the speed and $\mathrm{Y}$-axis is ratio of broadcasting nodes. 


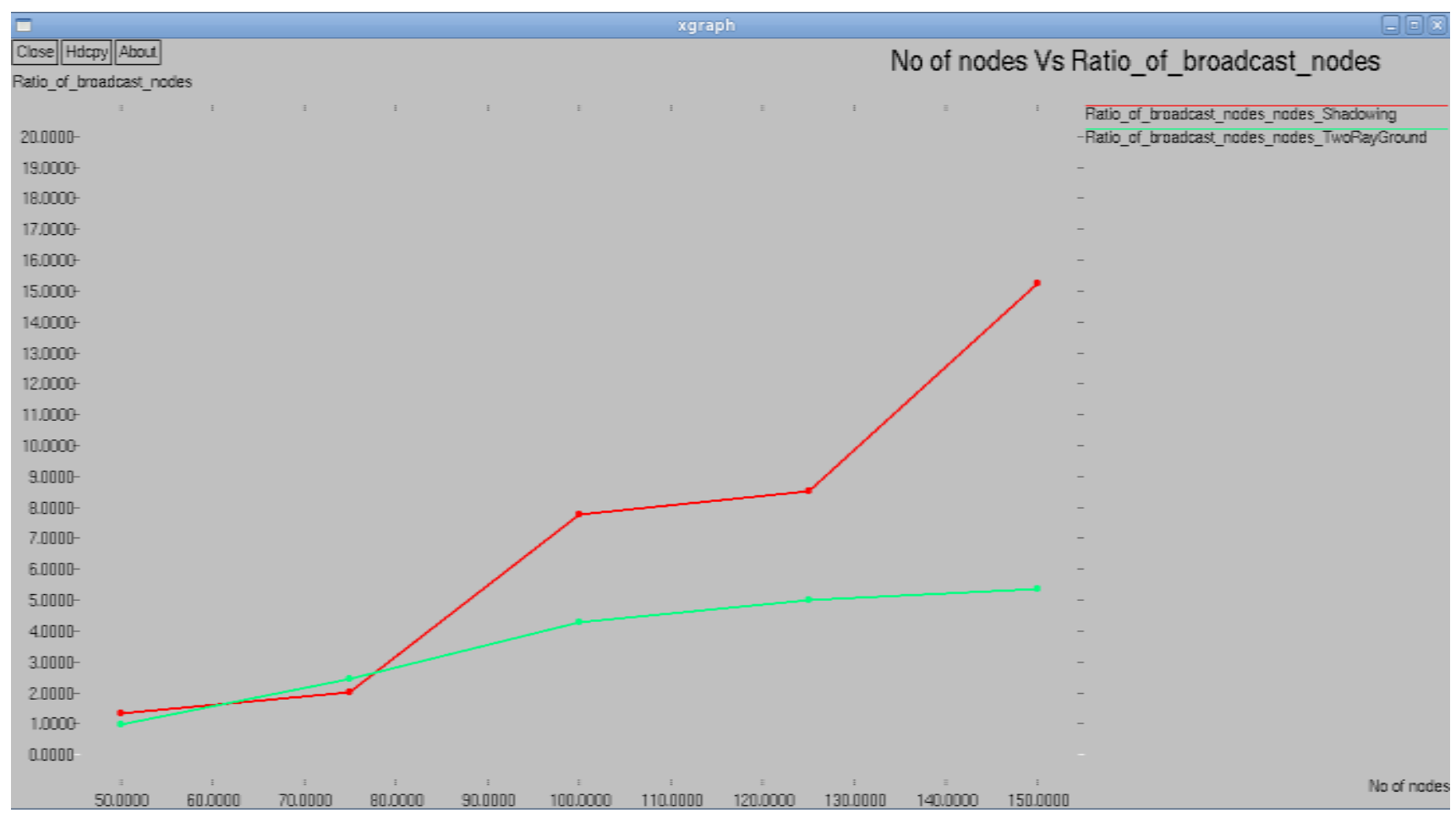

Fig2: Ratio of broadcasting nodes Vs total number of nodes

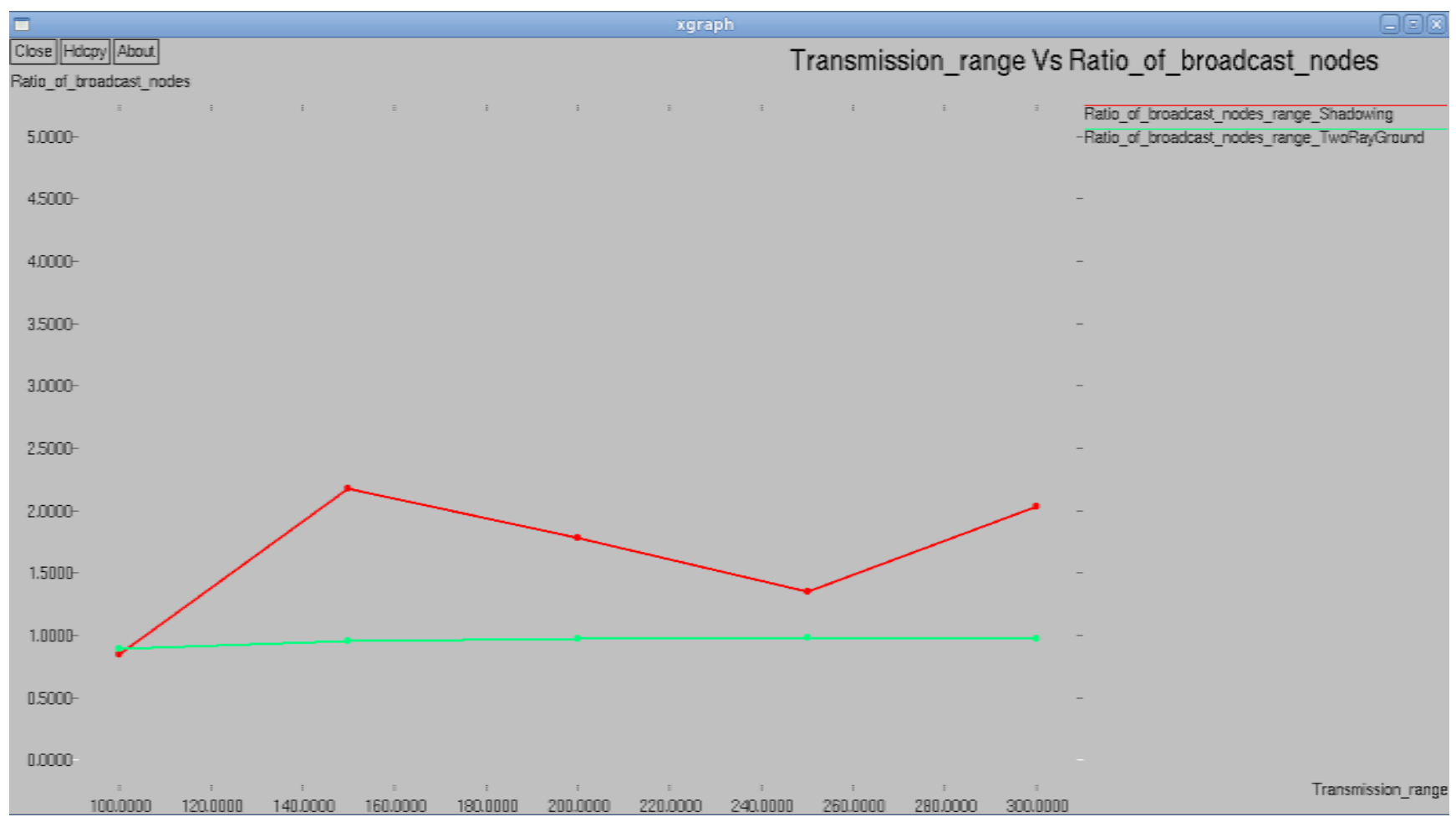

Fig 3: Ratio of broadcasting nodes versus transmission range 


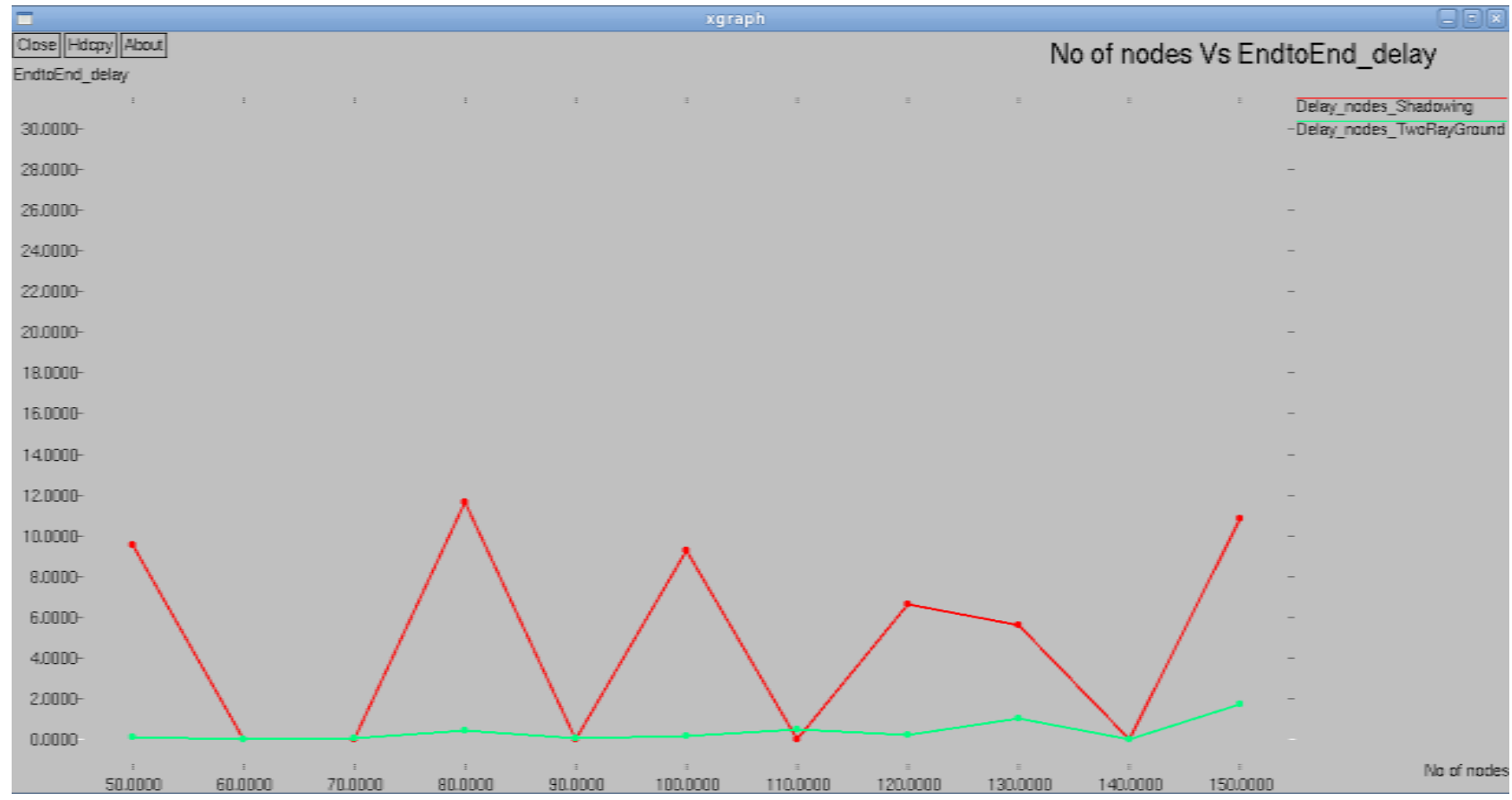

Fig 4: Average delay versus total number of nodes

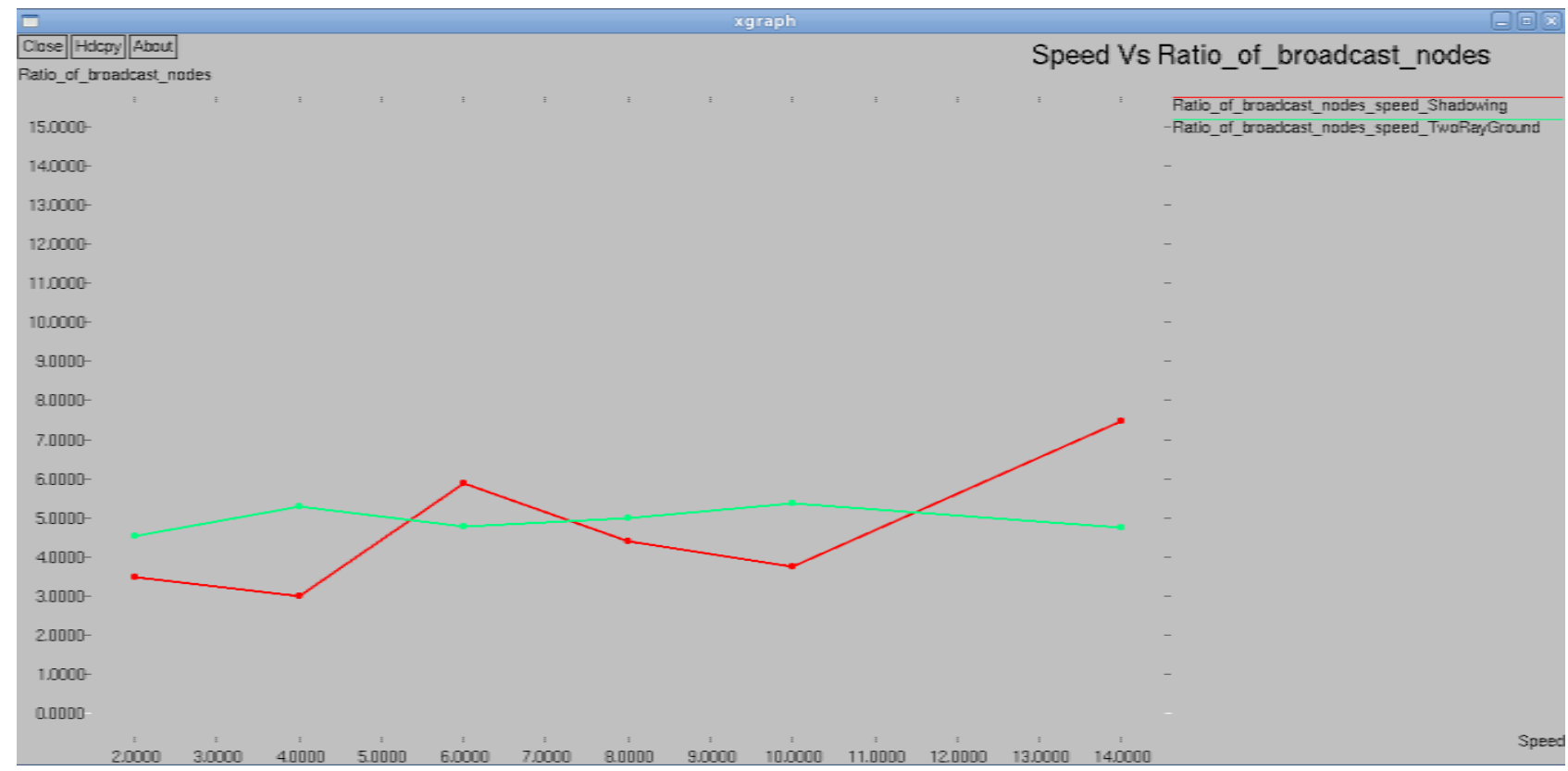

Fig 5: Ratio of broadcasting nodes versus maximum speed

Table 2: Performance analysis of different parameters

\begin{tabular}{|c|c|c|c|c|}
\hline Parameter & $\begin{array}{c}\text { Ratio of } \\
\text { broadcasting }\end{array}$ & $\begin{array}{c}\text { Transmission } \\
\text { range }\end{array}$ & $\begin{array}{c}\text { Average } \\
\text { delay }\end{array}$ & Max speed \\
\hline Shadowing & Increases & Increases & Increases & Increases \\
\hline Two-ray ground & Decreases & Decreases & Decreases & Increases \\
\hline
\end{tabular}




\section{CONCLUSION}

Local broadcast algorithms based on static approach can't guarantee good approximation factor if exact position of node is not available but some application it may not be possible to having exact position information of node. For that purpose we have presented dynamic approach in this approach exact position of node is not necessary we can easily achieve good approximation factor to their optimum solution. In this approach number of transmission rate is reduced to achieve optimum solution. This approach having number of advantages such as

- Avoid forwarding/rebroadcasting a message.

- Full delivery and constant approximation to the optimum solution.

- Calculate Maximum and Minimum transmission range of the nodes in the network.

This approach uses 2-hop hybrid algorithm for information exchange between nodes.

For future plan is to extend the performance of our algorithm under different scenarios. Dynamic approach can be extended to the case where the nodes have different transmission ranges. In this case, it can be proved that the algorithm guarantees a constant approximation. Also For future work, the throughput can be improved and battery level should be minimized to broadcast the message. Avoid copy of message while transferring the message to avoid the corruption of message.

\section{REFERENCES}

[1] S. Ni, Y. Tseng, Y. Chen, and J. Sheu, "The Broadcast Storm Problem in a Mobile Ad Hoc Network," Proc. ACM MobiCom, pp. 151-162, 1999.

[2] D.Y. Sassoon and A. Schiper, "Probabilistic Broadcast for Flooding in Wireless Mobile Ad Hoc Networks," Proc. IEEE Wireless Comm. and Networking Conf., pp. 1124-1130, 2003.

[3] H. Liu, P. Wan, X. Jia, X. Liu, and F. Yao, "Efficient Flooding Scheme Based on 1-Hop Information in Mobile Ad Hoc Networks,” Proc. IEEE INFOCOM, 2006.

[4] M. Khabbazian and V.K. Bhargava, "Efficient Broadcasting in Mobile Ad Hoc Networks," IEEE Trans. Mobile Computing, vol. 8, no. 2, pp. 231-245, Feb. 2009.

[5] W. Peng and X. Lu, "On the Reduction of Broadcast Redundancy in Mobile Ad Hoc Networks," Proc. ACM MobiHoc, pp. 129-2000.
[6] Y. CAI, K. Hua, and A. Phillips, "Leveraging 1-Hop Neighborhood Knowledge for Efficient Flooding in Wireless Ad Hoc Networks," Proc. IEEE Int'l Performance, Computing, and Comm. Conf. (IPCCC'05), pp. 347-354, 2005.

[7] M. Khabbazian and V.K. Bhargava, "Localized Broadcasting with Guaranteed Delivery and Bounded Transmission Redundancy," IEEE Trans. Computers, vol. 57, no. 8, pp. 1072-1086, Aug. 2008.

[8] J. Wu and F. Dai, "Broadcasting in Ad Hoc Networks Based on Self-Pruning," Proc. IEEE INFOCOM, pp. 2240-2250, 2003

[9] J. Wu and W. Lou, "Forward-Node-Set-Based Broadcast in Clustered Mobile Ad Hoc Networks," Wireless Comm. and Mobile Computing, vol. 3, no. 2, pp. 155173, 2003.

[10] J. Wu and F. Dai, "A Generic Distributed Broadcast Scheme in Ad Hoc Wireless Networks," IEEE Trans. Computers, vol. 53, no. 10, pp. 1343-1354, Oct. 2004.

[11] Z. Haas, J. Halpern, and L. Li, "Gossip-Based Ad Hoc Routing,’Proc. IEEE INFOCOM, pp. 1707-1716, 2002.

[12] J. Wu, W. Lou, and F. Dai, "Extended Multipoint Relays to Determine Connected Dominating Sets in Manets," IEEE Trans. Computers, vol. 55, no. 3, pp. 334-347, Mar. 2006.

[13] Y. Chen and J.L. Welch, "Location-Based Broadcasting for Dense Mobile Ad Hoc Networks," Proc. ACM Int'l Symp. Modeling, Analysis and Simulation of Wireless and Mobile Systems, pp. 63-70, 2005.

[14] I. Stojmenovic, M. Seddigh, and J. Zunic, "Dominating Sets and Neighbor Elimination-Based Broadcasting Algorithms in Wireless Networks," IEEE Trans. Parallel and Distributed Systems, vol. 13, no. 1, pp. 14-25, Jan. 2002.

[15] A. Keshavarz-Haddad, V. Ribeiro, and R. Riedi, "DRB and DCCB: Efficient and Robust Dynamic Broadcast for Ad Hoc and Sensor Networks," Proc. IEEE Fourth Ann. Comm. Soc. Conf. Sensor, Mesh and Ad Hoc Comm and Networks (SECON '07), pp. 253-262, 2007.

[16] P. Wan, K. Alzoubi, and O. Frieder, "Distributed Construction of Connected Dominating Set in wireless Ad hoc Networks," Proc. IEEE INFOCOM, vol. 3, pp. $1597-1604,2002$ 